\title{
Effects of deep lamellar keratoplasty on severe necrotizing stromal keratitis.
}

\author{
Xiaoru Shi, Yang Liu, Hui Jia, Lei Liu, Chunmei Wang, Aipeng Li ${ }^{*}$ \\ Department of Ophthalmology, the First Hospital of Jilin University, Changchun, Jilin, PR China
}

\begin{abstract}
Objective: This study is to investigate the application of deep lamellar keratoplasty (DLKP) in the treatment of necrotizing stromal keratitis (NSK).

Methods: Corneal confocal microscopy and histopathological examination were performed before surgery. DLKP and penetrating keratoplasty (PKP) were performed on 14 and 20 NSK subjects, respectively, followed by proper drug administration. In the follow-up period, the pathological features were evaluated and compared.

Results: Significant corneal neovascularization and inflammatory cell infiltration were observed in NSK patients before surgery. After proper drug treatments, PKP and DLKP were performed. In the followup period, the visual acuity was elevated in the PKP and DLKP groups at $1 \mathrm{y}$ after surgery. Moreover, corneal opacity was observed in 2 corneal grafts in the PKP group, while all the corneal grafts were transparent in the DLKP group. Furthermore, compared with the PKP group, corneal endothelial cell numbers at 6 and 12 months after surgery were significantly higher in the DLKP group. On the other hand, there were 2 eyes reporting virus recurrence in the PKP group, while no virus recurrence was reported in the DLKP group. Moreover, 4 eyes were accompanied with neovascularization at the corneal graft edge in the PKP group, while there was only 1 eye associated with neovascularization in the DLKP group. Furthermore, 5 cases in the PKP group reported graft rejection, while no rejection case was reported for DLKP.

Conclusion: DLKP provides superior outcomes to PKP in treating NSK, which might be an ideal alternative option for the disease treatment.
\end{abstract}

Keywords: Necrotizing stromal keratitis (NSK), Deep lamellar keratoplasty (DLKP), Penetrating keratoplasty (PKP). Accepted on November 15, 2017

\section{Introduction}

Herpes simplex virus keratitis (HSK) is the most common type of corneal inflammation, ranking the first in causing blindness among corneal diseases, with more than 10 million patients throughout the world [1]. HSK is clinically characterized by continuous relapses, however, due to lack of effective treatments, recurrent episodes would aggravate the corneal opacity, eventually leading to blindness. Necrotizing stromal keratitis (NSK) is one of the most serious types of HSK, commonly accompanied by yellow-white necrotic infiltration, with extremely poor prognosis. NSK often induces stromal neovascularization, stretching from the peripheral cornea toward the central area, which might eventually lead to corneal ulcers, thinning, and even perforation.

It has been accepted that the penetrating keratoplasty (PKP) is currently the only effective treatment for severe NSK with impending perforation, which can effectively control inflammation and improve visual acuity [2]. With appropriate administration of glucocorticoids and antiviral drugs, satisfactory therapeutic outcomes would be expected for PKP. However, surgical failure may still occur because of the elevated rejection rate resulting from corneal neovascularization and inflammation. Moreover, the virus recurrence after surgery and the consequent immune response might increase the graft opacity, especially the endothelial opacity [3-5].

Deep lamellar keratoplasty (DLKP), or deep anterior lamellar keratoplasty, is referred to as a surgical method that completely removes the damaged corneal tissue, to directly expose the Descemet's membrane. With the combination of air injection and tissue dissection, an ideal smooth planting bed would be obtained close to the Descemet's membrane [6]. Compared with traditional PKP, the rejection rate of DLKP is relatively low, with less post-operative complications, declined corneal endothelial cell loss, improved visual acuity, and less stringent requirement for donor cornea [7-9]. In recent years, DLKP has been rapidly developed and widely used in the treatment of various corneal diseases. However, the application of DLKP in the treatment of severe NSK has not yet been fully reported.

In this study, the application of DLKP in the treatment of NSK was investigated, in comparison with PKP. Totally 14 subjects (16 eyes) received DLKP, while PKP was performed on 20 subjects (21 eyes). These NSK patients were subjected to preoperative corneal confocal microscopy detection and 
pathological examination. After surgery, patients were subjected to proper drug treatments with antiviral drugs, immunosuppressants, and hormones. In the follow-up period, the treatment outcomes were analyzed and compared.

\section{Materials and Methods}

\section{Study subjects and grouping}

Totally 34 patients with NSK (37 eyes) were included in this study, who were admitted to our hospital from June 2008 to June 2010. These patients had been suffering from recurrent red eye, photophobia, lacrimation, accompanied with declined visual acuity, for 3-11 y. Uneven stromal opacity, dissolution, and ulcers, were observed in the corneal center, with a diameter of 7.0-11.0 $\mathrm{mm}$. Pre-operative corneal confocal microscopy was performed for each subject, to exclude fungal and Acanthamoeba keratitis. Antiviral drugs, immunosuppressants (cyclosporine A), and hormones were given when obvious neovascularization and inflammatory cell infiltration were observed. No vitreous opacity was examined with B-ultrasound, and there was also no retinal detachment. Clinical stability should last for $6 \mathrm{~m}$ before surgical operation. These NSK cases were divided into the PKP and DLKP groups. The PKP group contained 20 subjects (21 eyes), 9 males ( 9 eyes) and 11 females (12 eyes); and the DLKP group included 14 subjects ( 16 eyes), 6 males ( 6 eyes) and 8 females (10 eyes), with a mean age of $52 \mathrm{y}$ (ranging from 42 to $69 \mathrm{y}$ ).

For the vision detection, there were 7 cases ( 7 eyes) of HM/20 $\mathrm{cm}, 6$ cases ( 6 eyes) of $\mathrm{FC} / 20 \mathrm{~cm}$, and 7 cases ( 8 eyes) with 0.01-0.02 in the PKP group, while the DLKP group included 8 cases ( 8 eyes) of HM/20 cm, 4 cases ( 4 eyes) of FC/20 cm, and 2 cases (4 eyes) with 0.01-0.02. Prior written and informed consent were obtained from every patient and the study was approved by the ethics review board of Jilin University.

\section{Donor cornea acquisition}

All the donor cornea materials were collected from eyes obtained immediately after death (donor ages ranging from 20 to $40 \mathrm{y}$ ), according to a formal method [10]. These materials were kept in wet environment within $6 \mathrm{~h}$ after collection. Infectious diseases and other corneal transplantation contraindications were excluded.

\section{Deep lamellar keratoplasty (DLKP)}

For the DLKP procedures, topical ocular anesthesia was performed with $2 \%$ lidocaine and $0.5 \%$ bupivacaine (1:1). Eyelid was opened, and the upper and lower rectus muscles were sutured to fix the eyeball. Impaired cornea was drilled no more than $1 / 2$ in depth, using five $7.0 \mathrm{~mm}$, eight $7.5 \mathrm{~mm}$, and three $7.75 \mathrm{~mm}$ trephines. Impaired corneal stroma was cut layer by layer with a microscopic scalpel. When approaching the Descemet's membrane, the surface was washed with normal saline. The residue stromal fibers were lifted, and the Descemet's membrane was isolated from the stroma along the sectional edge with scalpel. Healthy corneal grafts with diameter of more than $0.25 \mathrm{~mm}$ were used for surgery. The whole corneal cortex was tore off, and placed on the planting bed. The interrupted nodules were sutured up, and the eyes were bandaged after surgery. For PKP, conventional surgery was conducted using eight $7.0 \mathrm{~mm}$, nine $7.5 \mathrm{~mm}$, and four 7.75 $\mathrm{mm}$ trephines. All the surgical procedures were performed by the same surgeon. All the removed corneal tissue was subjected to the histopathological examination.

\section{Hematoxylin and eosin (HE) staining}

Corneal tissues were fixed in $4 \%$ formaldehyde $(\mathrm{pH} \mathrm{7.0)}$ for less than $24 \mathrm{~h}$. After paraffin embedding, these tissues were cut into $4 \mu \mathrm{m}$ sections. The sections were subjected to HE staining according to a previously published protocol [11].

\section{Post-operative drug treatments}

On the first day after surgery, the interlayer and planting bed edges in all cases were tightly closed, with normal intraocular pressure and no formation of double anterior chamber. After surgery, cyclosporine A eye drops (North China Pharmaceutical Co., Ltd., Shijiazhuang, Hebei, China) were administrated once per day for the first $3 \mathrm{~d}$, four times per day for the next $3 \mathrm{~m}$, and three times per day for the next half a year to a whole year. Tobramycin dexamethasone ophthalmic ointment (Alcon, Inc, Dallas, TX, USA) was given once per day for the first $3 \mathrm{~d}$, and three times per day for the next month. Fluorometholone (Santen Pharmaceutical Co., Ltd., Higashiyodogawa, Osaka, Japan) was then used three times per day for half a year. According to the patient's condition, the drug administration could be continued twice per day for another whole year. Ganciclovir ophthalmic gel (Ruilin, Shenzhen, Guangdong, China) was used once per day for the first $3 \mathrm{~d}$, and four times per day for the next month. Acyclovir (Hidragon, Qianjiang, Hubei, China) was intravenously administered $(500 \mathrm{mg})$ three times per day for 7-10 d. Prednisone (Tianjin Pacific Chemical \& Pharmaceutical Co., Ltd., Tianjin, China) was orally administered $(20 \mathrm{mg})$ once per day on the first week, $10 \mathrm{mg}$ once per day on the second week and $5 \mathrm{mg}$ once per day on the third week. Cyclosporine A capsules (North China Pharmaceutical Co., Ltd.) were orally administered $(5 \mathrm{mg} / \mathrm{kg})$ twice per day for $3 \mathrm{~m}$. The same drug administration was performed for both the PKP and DLKP groups.

\section{Post-operative examinations}

Corneal confocal microscopy and anterior segment OCT examination were performed for the PKP and DLKP groups, measuring the corneal endothelial cell number and detecting the healing process, at 6 and $12 \mathrm{~m}$ after surgery, respectively. The corneal endothelial cell number was counted manually by two individuals on the same images and calculated as the average number counted by these two individuals. Visual acuity, intraocular pressure, corneal neovascularization, virus recurrence, and graft rejection were determined and recorded. Corneal graft rejection was confirmed when the subject was accompanied with decreased visual acuity or corneal edema, 
with or without subcutaneous infiltration and anterior segment inflammation.

\section{Statistical analysis}

SAS 9.0 software was used for statistical analysis. The paired t-test and $\chi^{2}$ test were performed for group comparison. $\mathrm{P}<0.05$ was considered statistically significant.

\section{Results}

\section{Pre-operative corneal confocal microscopy detection and pathological examination}

Pre-operative corneal confocal microscopy detection and pathological examination were performed for the NSK patients. Corneal confocal microscopy showed that, the corneal basal cells displayed significantly abnormal morphology, with plenty of intercellular deposits. Corneal nerve fibers were thickened, with granular morphology, and significant alterations were observed in the corneal endothelial cells. Moreover, corneal neovascularization and inflammatory cell infiltration were also observed with corneal confocal microscopy in NSK (Figure 1).



Figure 1. Pre-operative corneal confocal microscopy detection of NSK. NSK was detected with corneal confocal microscopy. (A) Corneal neovascularization (arrow); (B) Obviously changed stromal cells (star) and numerous intercellular deposits (arrowhead).

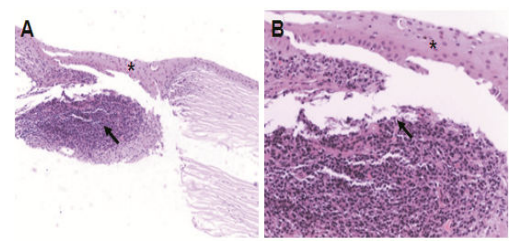

Figure 2. Histopathological detection of cornea with NSK. HE staining was performed to detect histological changes in cornea with $\operatorname{NSK}(A, 4 X ; B, 10 X)$. Stars indicated cornea thinning and epithelial atrophy, and arrows indicated inflammatory cell infiltration.

On the other hand, the pathological detection indicated obvious cornea thinning, epithelial atrophy, stromal layer atrophy, scarring, crack formation, and inflammatory cell infiltration, in these NSK cases, with occasionally observed lymphocyte plasma cell aggregation (Figure 2). These results suggest that typical pathological characteristics could be observed in these NSK subjects. After the treatments with antiviral drugs, immunosuppressants, and hormones, surgical operation was not performed until basically normal morphology was observed in the corneal basal cells, the intercellular deposits were absorbed, and the inflammatory cells were significantly decreased.

\section{Post-operative visual acuity, corneal graft transparency, and corneal endothelial cell numbers}

The visual acuity, corneal graft transparency, and corneal endothelial cell numbers in the NSK cases after surgery were investigated. The post-operative follow-up period lasted for 12-31 m, with an averaged period of $18.33 \mathrm{~m}$. Our results from the visual acuity detection showed that, at $1 \mathrm{y}$ after surgery, the visual acuities were elevated in both the PKP and DLKP groups, compared with before surgery. For the PKP group, there were 3 eyes with the visual acuity $\leq 0.1,10$ eyes between $0.2-0.4$, and 8 eyes $>0.5$. Visual acuity was slightly improved in the DLKP group (compared with the PKP group, $\mathrm{P}>0.05$ ), including 1 eye with the visual acuity $\leq 0.1,8$ eyes between $0.2-0.4$, and 7 eyes $>0.5$.

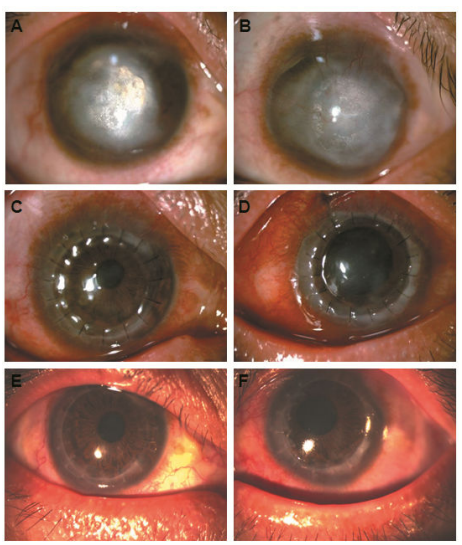

Figure 3. Comparison of examination images before and after DLKP. $(A, B)$ Images of right $(A)$ and left $(B)$ eyes with NSK before DLKP. Dramatic gray haze was observed in the corneal centers of both eyes, with uneven corneal thickness and obvious peripheral neovascularization. (C,D) Images of right $(C)$ and left (D) eyes with NSK at $1 \mathrm{w}$ after DLKP. Transparent corneal grafts were adhering to the planting beds. $(E, F)$ Images of right $(E)$ and left $(F)$ eyes with NSK at $17 \mathrm{~m}$ after DLKP. Corneal grafts remained transparent, which were healing well with the planting beds.

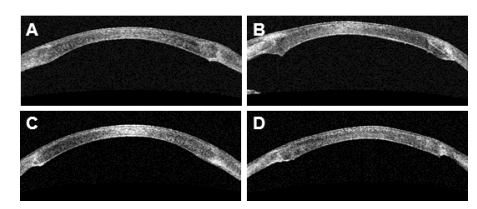

Figure 4. Anterior segment OCT examination before and after DLKP. OCT images of NSK cases at $1 \mathrm{w}(A$, right eye; $B$, left eye) and $17 \mathrm{~m}$ (C, right eye; $D$, left eye) after $D L K P$.

For the corneal graft transparency detection, opacity was observed in 2 corneal grafts in the PKP group, while all the corneal grafts remained transparent in the DLKP group $(\mathrm{P}>0.05)$. For DLKP, as shown in Figure 3, before the surgery, dramatic gray haze was observed in the corneal center, with uneven corneal thickness and obvious peripheral neovascularization. At $1 \mathrm{w}$ after DLKP, the transparent corneal grafts were adhering to the planting beds. At post-operative 17 $\mathrm{m}$, when the sutures had been removed, the corneal grafts remained transparent, which were healing well with the planting beds. The anterior segment OCT results at $1 \mathrm{w}$ and 17 
$\mathrm{m}$ after surgery in the DLKP group were shown in Figure 4. Transparent corneal grafts and satisfying interlayer healing process were observed.

In addition, the corneal endothelial cell counting showed that, the corneal endothelial cell numbers at 6 and $12 \mathrm{~m}$ after surgery in the DLKP groups were $2358 \pm 211$ and $2240 \pm 212$ cells $/ \mathrm{mm}^{2}$, respectively, which were significantly higher than those in the PKP group at the corresponding time points (1880 \pm 170 and $1643 \pm 197$ cells $/ \mathrm{mm} 2$ for 6 and 12 months after surgery, respectively; both $\mathrm{P}<0.01$ ). Taken together, these results suggest that, compared with PKP, DLKP provides increased visual acuity, satisfactory corneal graft transparency, and elevated corneal endothelial cell numbers after surgery in NSK cases.

\section{Post-operative complications and treatments}

Post-operative complications were analyzed and compared for PKP and DLKP. After the surgery, in the PKP group, 4 cases reported high intraocular pressure (IOP), three of which recovered to normal after treatment with IOP-lowering drugs, while the other case was alleviated after trabeculectomy. In the DLKP group, there was only 1 eye reporting high IOP due to the air injection into anterior chamber, which lasted for $2 \mathrm{~d}$. When the air was absorbed, the IOP recovered to normal level. Moreover, after the surgery, there were 4 cases of synechia in the PKP group, while there was 1 eye accompanied with mild synechia in the DLKP group. During the follow-up period, there were 2 eyes reporting virus recurrence in the PKP group, while no virus recurrences were reported in the DLKP group. Moreover, 4 eyes were accompanied with neovascularization at corneal graft edge in the PKP group, while there was only 1 eye associated with neovascularization in the DLKP group $(\mathrm{P}>0.05)$.

In addition, 5 cases in the PKP group reported graft rejection, while no immune rejection was reported in the DLKP group $(\mathrm{P}<0.05)$. These 5 rejection cases in the PKP group occurred at $3,6,10,16$, and $19 \mathrm{~m}$ after surgery, respectively, and all these cases were associated with numerous neovascularization and inflammatory cell infiltration as detected by pre-operative corneal confocal microscopy detection and pathological examination. After the treatments with corticosteroids and cyclosporine, three cases became transparent, while the remaining corneal grafts were turbid due to the corneal endothelial cell damage. Taken together, these results suggest that, compared with PKP, DLKP resulted in fewer complications after surgery, with reduced synechia, no virus recurrence and graft rejection report, after surgery, in NSK patients.

\section{Discussion}

Necrotizing stromal keratitis (NSK) is induced by the recurrent attacks of discoid or dendritic keratitis, which results from the infection of herpes simplex virus in corneal stromal cells $[12,13]$. NSK is clinically characterized by delayed hypersensitivity, including corneal neovascularization and severe inflammatory cell infiltration [14]. NSK might result in descemetocele, hypopyon, corneal perforation, and even mixed infection, with extremely poor prognosis. For NSK with impending perforation, PKP has been accepted as the only effective therapeutic treatment for a long time [2]. However, the higher rejection and virus recurrence rates have been also reported following PKP. In this study, based on the preoperative corneal confocal microscopy and pathological examination, as well as the post-operative rational drug administration, PKP and DLKP were performed on 20 NSK cases (21 eyes) and 14 cases (16 eyes), respectively. Our results suggest that, compared with PKP, DLKP could provide more satisfactory outcomes in the treatment of NSK.

In this study, 14 NSK patients (16 eyes) were subjected to DLKP. In the follow-up period, there were no reports about virus recurrence and graft rejection, while 2 cases of virus recurrence were reported in the PKP group. In a previous study from Sarnicola et al. [15], DLKP was performed on 52 subjects with viral keratitis. In the one-year follow-up period, $5 \%$ of these patients suffered from virus recurrence, and 33\% were associated with corneal graft rejection in the three-year followup period [16]. In another report from Wang et al. [17], 42 HSK cases were subjected to DLKP, and the virus recurrence rates for active and resting HSK after DLKP treatment were $18.2 \%$ and $9.5 \%$, respectively. Virus recurrence and graft rejection are the main risk factors for DLKP in the treatment of viral keratitis. However, the viral keratitis typing was not specifically focused on in the above studies. In this study, the most commonly seen viral keratitis, i.e., NSK, was investigated. The main mechanism for NSK relapse lies in the cell-mediated immune response induced by the virus antigen accumulated in the corneal stroma, and the active infection of herpes simplex virus in the stroma might not be the direct cause [18]. Because the virus antigen was accumulated in the corneal stroma, rather than in the endothelial layer or the Descemet's membrane, DLKP (which directly reached the Descemet's membrane) could provide more satisfactory outcomes concerning the virus recurrence compared with PKP.

In a previous study from Roni et al. [19], DLKP was performed on 62 NSK cases, $81 \%$ of which were in the clinically stable status. Pathological detection confirmed that $74 \%$ of these cases were associated with active corneal inflammation, which would be a high risk factor for corneal graft rejection. Therefore, pre-operative examination of corneal confocal microscopy is very important for patients with clinically stable viral keratitis. In this study, corneal confocal microscopy indicated numerous neovascularization and inflammatory cell infiltration. After proper treatments with antiviral drugs, immunosuppressants, and hormones, appropriate timing for surgery should be determined only if the neovascularization and inflammatory cell infiltration were obviously alleviated. In this study, our results showed that all the cases reporting virus recurrence and graft rejection after surgery were associated with inflammation in the pre-operative corneal confocal microscopy detection and pathological examination. Moreover, in some of the subjects with neovascularization before surgery, no neovascularization was 
observed in the post-operative examination. This phenomenon might be explained by the fact that neovascularization happened in the peripheral region, and it might depend on the position and orientation of histological sections. Based on these findings, we hypothesize that the subjects with severe inflammatory cell infiltration are prone to the corneal graft rejection, which should be given timely detection and appropriate treatments. In clinic, inflammation in NSK is difficult to deal with, especially with numerous neovascularization. In this study, satisfactory outcomes were obtained for the DLKP group, which might result from the proper surgical timing based on the pre-operative corneal confocal microscopy, thorough lesion debridement (reaching the Descemet's membrane), and appropriate drug administration according to the pathological detection. However, due to the limited subject numbers and follow-up period, further studies are still needed to investigate the longterm effects of DLKP.

Neovascularization is a risk factor for corneal graft rejection and virus recurrence [4]. In this study, 1 case reported corneal neovascularization after surgery in the DLKP group, while there were 4 cases of neovascularization in the PKP group. On the other hand, insufficient corneal endothelial cells after surgery might lead to corneal endothelial decompensation and corneal graft failure. Our results showed that, the corneal endothelial cell numbers at 6 and $12 \mathrm{~m}$ after surgery in the DLKP were both significantly higher than the PKP group, indicating the superiority of DLKP to PKP in the treatment of NSK. However, DLKP is in fact a rather difficult surgical operation, requiring more experience and sophisticated skills. Proper surgical timing is particularly important to increase the surgical safety and accuracy, which could be determined according to the pre-operative examinations. Appropriate drug administration should be given to the NSK subjects with severe abnormal corneal endothelial cells before they were ready for the DLKP operation. This study has some limitations. First, the sample size was relatively small. Second, sample size calculation was not performed. Third, the follow-up period was relatively short and long-term efficacy of DLKP was not observed.

\section{Conclusion}

In conclusion, our results showed that, compared with PKP, DLKP provided more satisfactory outcomes in the treatment of NSK. DLKP resulted in fewer complications after surgery, reduced iris reaction, restored corneal thickness, increased corneal resilience, decreased corneal graft rejection, and elevated corneal endothelial cells. These findings suggest that DLKP might be an ideal alternative option for the treatment of NSK in clinic.

\section{Acknowledgement}

This study was supported by First Hospital of Jilin University Hospital.

\section{References}

1. Farooq AV, Shukla D. Herpes simplex epithelial and stromal keratitis: an epidemiologic update. Surv Ophthalmol 2012; 57: 448-462.

2. Hogan M. Corneal transplantation in the treatment of herpetic disease of the cornea. Am J Ophthalmol 1957; 43: 147-160.

3. Cobo L, Coster D, Rice N, Jones B. Prognosis and management of corneal transplantation for herpetic keratitis. Arch Ophthalmol 1980; 98: 1755-1759.

4. Garcia D, Shtein R, Musch D, Elner V. Herpes simplex virus keratitis: histopathologic neovascularization and corneal allograft failure. Cornea 2009; 28: 963-965.

5. Lomholt J, Baggesen K, Ehlers N. Recurrence and rejection rates following corneal transplantation for herpes simplex keratitis. Acta Ophthalmol Scand 1995; 73: 29-32.

6. Archila E. Deep lamellar keratoplasty dissection of host tissue with intrastromal air injection. Cornea 1984; 3: 217-218.

7. Coombes A, Kirwan J, Rostron C. Deep lamellar keratoplasty with lyophilised tissue in the management of keratoconus. Br J Ophthalmol 2001; 85: 788-791.

8. Li J, Yu L, Deng Z, Wang L, Sun L, Ma H, Chen W. Deep anterior lamellar keratoplasty using acellular corneal tissue for prevention of allograft rejection in high-risk corneas. Am J Ophthalmol 2011; 152: 762-770.

9. Shimazaki J, Shimmura S, Ishioka M, Tsubota K. Randomized clinical trial of deep lamellar keratoplasty vs penetrating keratoplasty. Am J Ophthalmol 2002; 134: 159-165.

10. Lass JH, Szczotka-Flynn LB, Ayala AR, Benetz BA, Gal RL, Aldave AJ, Corrigan MM, Dunn SP, McCall TL, Pramanik S, Rosenwasser GO, Ross KW, Terry MA, Verdier DD; Writing Committee for the Cornea Preservation Time Study Group. Cornea preservation time study: Methods and potential impact on the cornea donor pool in the united states. Cornea 2015; 34: 601-608.

11. http://www.ihcworld.com/_protocols/special_stains/ h\&e_ellis.htm

12. Müller RT, Pourmirzaie R, Pavan-Langston D, Cavalcanti BM, Aggarwal S, Colón C, Jamali A, Cruzat A, Hamrah P. In vivo confocal microscopy demonstrates bilateral loss of endothelial cells in unilateral herpes simplex keratitis. Invest Ophthalmol Vis Sci 2015; 56: 4899-4906.

13. Yokogawa H, Kobayashi A, Mori N, Sugiyama K. Mapping of dendritic lesions in patients with herpes simplex keratitis using in vivo confocal microscopy. Clin Ophthalmol 2015; 24: 1771-1777.

14. Rogge M, Yin XT, Godfrey L, Lakireddy P, Potter CA, Del Rosso CR, Stuart PM. Therapeutic use of soluble fas ligand ameliorates acute and recurrent herpetic stromal keratitis in mice. Invest Ophthalmol Vis Sci 2015; 56: 6377-6386.

15. Sarnicola V, Toro P. Deep anterior lamellar keratoplasty in herpes simplex corneal opacities. Cornea 2010; 29: 60-64. 
16. Awan M, Roberts F, Hegarty B, Ramaesh K. The outcome of deep anterior lamellar keratoplasty in herpes simplex virus-related corneal scarring, complications and graft survival. Br J Ophthalmol 2010; 94: 1300-1303.

17. Wang J, Zhao G, Xie L, Chen M, Zhao J. Therapeutic effect of deep anterior lamellar keratoplasty for active or quiescent herpetic stromal keratitis. Graefes Arch Clin Exp Ophthalmol 2012; 250: 1187-1194.

18. Zhao G, Chen M, Liu T, Sun SY, Zhao J, Xie LX. Association of HSV-1 antigen distribution in the cornea with clinical characteristics of herpetic stromal keratitis. Eur J Ophthalmol 2012; 22: 40-45.
19. Shtein R, Garcia D, Musch D, Elner V. Herpes simplex virus keratitis: histopathologic inflammation and corneal allograft rejection. Ophthalmology 2009; 116: 1301-1305.

\section{*Correspondence to}

Aipeng Li

Department of Ophthalmology

The First Hospital of Jilin University

PR China 